\title{
Scanning Surface Potential Microscopy of Spores on Planar Surfaces
}

\author{
I. Lee, ${ }^{1}$ E. Chung, ${ }^{2}$ H. Kweon, ${ }^{2}$ S. Yiacoumi, ${ }^{2}$ D.C. Joy, ${ }^{1,3}$ A.V. Palumbo, ${ }^{3}$ and C. Tsouris ${ }^{2,3}$ \\ ${ }^{1}$ Department of Electrical Engineering \& Computer Science, University of Tennessee, Knoxville, \\ TN 37996-2100 \\ ${ }^{2}$ School of Civil \& Environmental Engineering, Georgia Institute of Technology, Atlanta, GA 30332 \\ ${ }^{3}$ Oak Ridge National Laboratory, Oak Ridge, TN 37831
}

We have investigated the electrostatic potential of Bacillus thuringiensis (BT) spores on various substrates by using our combined atomic force microscopy (AFM) and scanning surface potential microscopy (SSPM) technique. SSPM, also known as Kelvin probe force microscopy (KPFM), has been previously used to map the surface morphology and the corresponding local electrostatic potential of Photosystem I reaction centers [1], air-liquid interface [2], proteoliposomes [3], and bacteriorhodopsin [4] in nanometer resolution.

The adhesion of Bacillus anthracis - the cause of anthrax and a likely biological threat - to solid surfaces is important for cleanup after an accidental or deliberate release. However, directly studying $B$. anthracis spores with advanced instrumentation is problematic due to safety concerns. As a first step, we are examining adhesion of BT, which is a closely related species and is often used as a simulant to study $B$ anthracis. Bacterial spore adhesion to inert surfaces is generally believed to involve surface hydrophobicity [5-6], surface polarity [7], surface electrical charge [8-9], and van der Waals force.

The BT spores were imaged with the side-by-side AFM and SSPM, as illustrated in Fig. 1A and 1B. Both the size and surface potential were measured by section analysis. The SSPM surface potential section analysis is shown in Fig. 2. At a relative humidity $\mathrm{RH}=64 \%$, the surface potential between $\mathrm{BT}$ and substrate is $-1.50 \mathrm{~V}$, and the substrate potential is $-0.57 \mathrm{~V}$. Table 1 shows the BT and substrate potentials at a $\mathrm{RH}$ ranging from $9 \%$ to $64 \%$. The shape and dimension of the spore were confirmed by scanning transmission electron microscopy (STEM) and scanning electron microscopy (SEM). We did not locate any spores on mica surface. This is because the surface potential of mica is extremely high, $-4.8 \mathrm{~V}$ at $\mathrm{RH} 15 \%$, leading to a strong electrostatic repulsion between the mica surface and the spore. For the same reason, the adhesion force measurement between the spore and mica by AFM is zero. Our SSPM results are thus consistent with adhesion force measurements.

\section{References}

[1] I. Lee et al., J. Phys. Chem. B 104 (2000) 2439.

[2] I. Lee et al., J. Phys. Chem. B 107 (2003) 14225.

[3] T. Kuritz et al., IEEE Trans. on NanoBiosci. 4, No. 2 (2005) 196.

[4] I. Lee et al., J. Phys. Chem. B 110 (2006)10982.

[5] U. Husmark and U. Ronner, Biofouling 5 (1992) 335.

[6] U. Hüsmark and U. Ronner, Biofouling 7 (1993) 57.

[7] L. Bouiange-Petermann et al., J. Adhes. Sci. Technol. 7 (1993) 221.

[8] U. Husmark and U. Ronner, J. Appl. Bacteriol. 69 (1990) 557.

[9] S. H. Flint et al., J. Food Eng. 43 (2000) 235. 


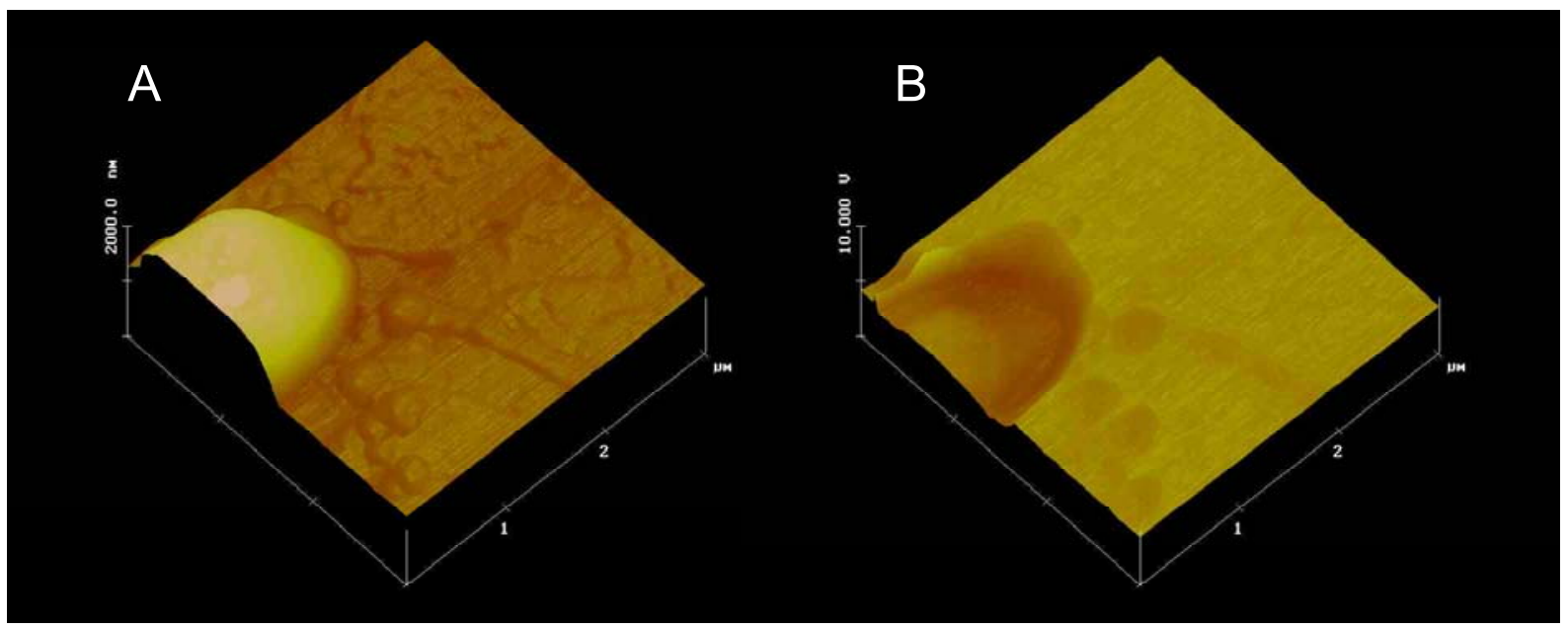

Fig. 1. Images of a spore at $\mathrm{RH}=64 \%$ on a silica surface. A: Tapping-mode AFM image of topography. B: SSPM image of electrostatic surface potential.

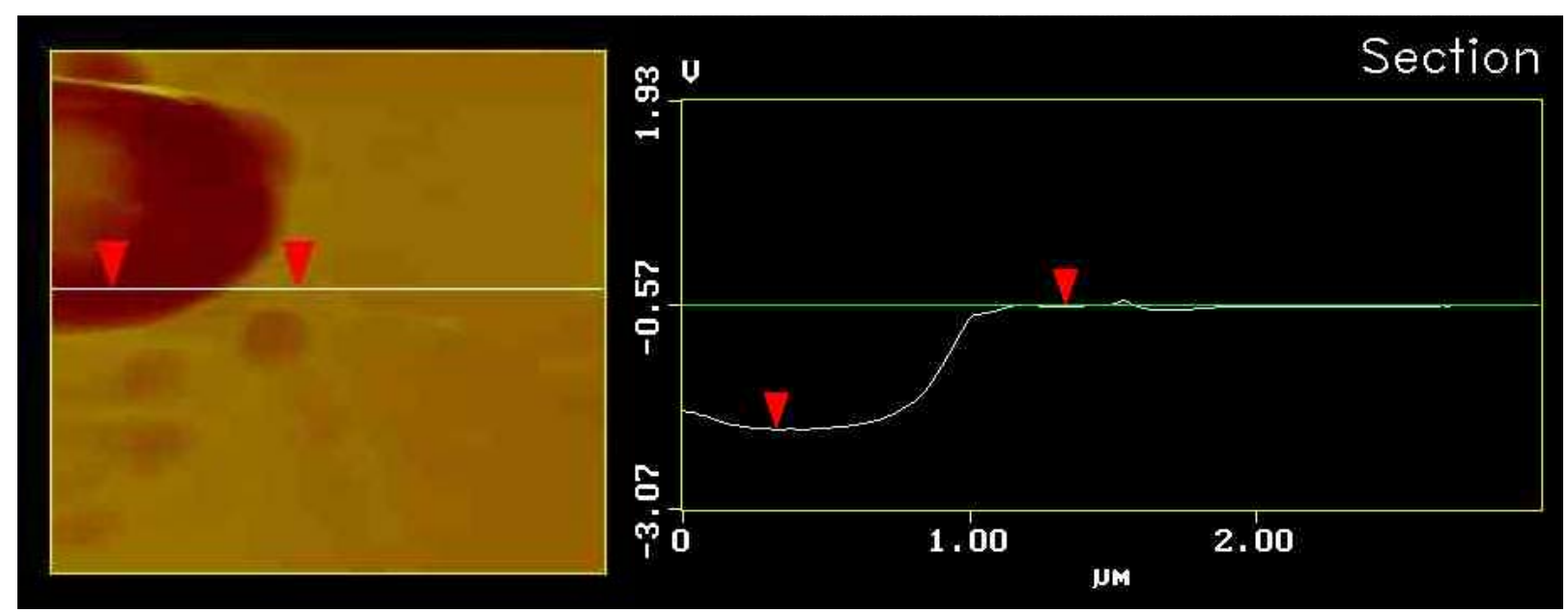

Fig. 2. SSPM section analysis at $\mathrm{RH}=64 \%$. The surface potential between $\mathrm{BT}$ and substrate is $-1.50 \mathrm{~V}$, and the substrate potential is $-0.57 \mathrm{~V}$.

TABLE 1. Surface electric potential of BT and substrate at various humidity values.

\begin{tabular}{ccc}
\hline RH $(\%)$ & BT Potential (V) & Substrate Potential (V) \\
\hline 9 & -1.65 & -0.21 \\
20 & -1.48 & -0.80 \\
30 & -1.44 & -1.02 \\
46 & -1.48 & -0.84 \\
64 & -1.50 & -0.57 \\
\hline
\end{tabular}

